

\title{
Some insights into the effect of maintenance quality for a protection system
}

\author{
M.D. Berrade, P.A. Scarf, and C.A.V. Cavalcante, Member, IEEE
}

\begin{abstract}
This paper considers an inspection and preventive replacement policy for a one-component protection or cold standby system. Inspection is imperfect, and subject to false positives and negatives; preventive replacement may also be of poor quality. We determine conditions relating to the quality of the inspection and preventive replacement under which a maintained system would not benefit from the execution of inspections and preventive maintenance. We present examples with decreasing failure rate component lifetimes in which preventive replacement is cost-optimal, contrary to the classic policy. Such cases arise when inspections do not necessarily detect the failed state.
\end{abstract}

Index Terms-Inspection, replacement, mixture, cold standby, preparedness, optimum policy

\section{ABBREVIATIONS}

$D F R \quad$ Decreasing failure rate

$I F R \quad$ Increasing failure rate

\section{NOTATION}

$X \quad$ component lifetime

$R(x) \quad$ reliability or survival function of $X$

$\mu=\mathrm{E}[\mathrm{X}] \quad$ expected component lifetime

$T$ inspection interval

$\alpha \quad$ probability of a false positive inspection

$\beta \quad$ probability of a false negative inspection

$M \quad$ maximum number of inspections until preven-

tive replacement.

$K_{1} \quad$ number of inspections previous to failure or a false positive or to preventive replacement whichever occurs first

$K_{2} \quad$ number of inspections after failure until its detection or preventive replacement whichever occurs first

$U$ uptime in a cycle

$D$ downtime in a cycle

$c_{0} \quad$ cost of inspection

$c_{m} \quad$ cost of a preventive replacement either at time

$M T$ or at a replacement at a false positive inspection

$c_{r} \quad$ cost of replacement at a true positive inspection

$c_{d} \quad$ cost-rate of unavailability

$c_{\text {ins }}$ expected cost due to all inspections in a cycle

$c_{\text {ren }} \quad$ expected cost derived from the replacement of the system

M.D. Berrade is with the Department of Statistics, School of Engineering and Arquitecture (EINA), University of Zaragoza, Zaragoza, 50018 Spain (email: berrade@unizar.es).

P.A. Scarf is with Salford Business School, University of Salford, Manchester, M5 4WT, U.K. (e-mail: p.a.scarf@salford.ac.uk).

C.A.V. Cavalcante is with the Department of Production Engineering, Federal University of Pernambuco, Recife-PE, 740-530, Brazil (e-mail: cristiano@ufpe.br).

\author{
$\tau$ length of a cycle \\ $C(\tau) \quad$ total cost of a cycle \\ $Q(T, M) \quad$ cost rate (the long run expected cost per \\ unit time)
}

\section{INTRODUCTION}

$\mathbf{T}$ HIS paper focuses on the quality of maintenance, and in particular on the quality of inspection. Inspection policies are typically used when failures are hidden, where a test or inspection is required to establish the state of the system. This hidden failure condition is the case for systems that are not in continuous operation but alternate between idle periods and periods of use. When a failure can occur during an idle period, if such a failure occurs and there is no regular inspection, the failure will remain undetected until there is an attempted use of the system.

Protection or preparedness systems and cold standby systems are typical examples of systems that operate in this way, and thus experience hidden failures. Apostolakis and Bansal [1] describe safety features of nuclear power plants consisting of redundant systems that are inactive until emergency conditions occur (e.g., pump failure in a cooling system). These systems undergo inspections at regular intervals to ensure their high availability. The consequences of poor quality maintenance, including poorly executed replacements or low quality spare parts [2], are reported by technology users or operators ( [3], [4]). In our paper here, we model the quality of inspections, and analyze when low quality has non-negligible consequences on both maintenance procedures and systems reliability.

Inspection policies for systems subject to hidden failures have been studied by many authors. A classic reference is Vaurio [5]. Recent work has considered multi-component systems (e.g [6]), and hard (revealed) and soft (unrevealed) failures of multi-component systems (e.g [7]). Models have been proposed when inspection and replacement downtimes are non-negligible (e.g. [8]).

Inspection policies with false positives or false negatives or both are analysed in [9]- [13]. Okumura et al. [9] present an inspection model based on the delay-time model. Human errors are considered in [1], and [10]. Gong [14] investigates a repetitive testing process where the testing equipment may leave the in-control state at random, resulting in different testing errors.

At a false positive event, inspection indicates that the system is failed when it is in fact not failed. At a false negative, inspection does not reveal an existing failure. In Berrade et al. [12], the effect of such imperfect inspection is further 
analysed by presenting two scenarios, one of which is related to the outsourcing of maintenance, and the other is related to maintenance performed in-house. In our paper, we analyze in detail the second scenario, and in particular we characterise circumstances in which false positives can lead to unnecessary replacements. We also implicitly consider circumstances in which false negatives do the same. This consideration is important because, if false negatives tend to occur with high probability, a typical response might be to do more inspection. Moreover, more inspection will lead to more false positives; and, where we have DFR component lifetimes, more inspection will lead to the possibility of more unnecessary replacements of good components by poor components. We model this low quality replacement by a mixture of two Weibull distributions (see e.g. [16], [20]). Cha and Finkelstein [17] also consider two ordered sub-populations with strong, and weak units respectively for a burn-in procedure.

When the population is heterogeneous, that is, strong and weak spares are mixed, failed components are replaced by new ones, which in turn can be weak or strong. However, in the case of imperfect inspections, another consequence emerges which is that strong, working components can be replaced by weak ones. Thus, an actual decrease of reliability can occur when heterogeneous components may undergo imperfect inspections.

Our paper analyzes this situation just described. Furthermore, whereas Ten Wolde and Ghobbar [15] for example state that increasing the inspection frequency increases the chance that a potential failure will be identified so that the additional cost (of more inspections) leads to improved reliability, this condition is not the case when the consequence of a false positive inspection is the possible replacement of a strong component by a weak one (Berrade et al. [13]). Therefore, in our view, it is necessary to consider both cost and system reliability when analyzing the effect of inspection errors and low quality maintenance on inspection policy, and we do so in our paper here.

In the next section, we describe our maintenance policy and its features, including the underlying component reliability model. We then determine conditions under which there exists a finite optimum inspection interval (Section 2.1). In so doing, we also quantify circumstances in which the quality of inspection and replacement is not sufficiently high for the benefit derived from inspections to compensate for their incurred cost. In this way, we provide conditions that relate to the quality of maintenance and spare parts under which a maintained system would not benefit from the execution of preventive maintenance. Proofs of our results are given in the Appendix. In Section 2.2, we further determine the operational reliability of the system. This knowledge may be useful when one wishes not only to consider a maintenance policy that is safety driven rather than cost driven (e.g. Flage et al. [18]), but also to consider the possibility that an increasing cost (of inspection) does not imply an increasing reliability. We then consider some numerical examples, the first of which presents a discrete mixture for the failure time distribution (Section 3.1), and the second a continuous mixture (Section 3.2). The examples provide additional insight about how the interaction between inspection errors and low quality maintenance cannot be neglected. We finish with a short discussion of the implications for the management of engineering services.

\section{MAINTENANCE POLICY}

Consider a system with failures that are detected only by inspection. The system is inspected periodically every $T$ units up to replacement. The system is replaced at a positive inspection, which is when inspection indicates the occurrence of a failure.

Testing may be imperfect. At an inspection, a false positive can occur. A false positive is the case when the inspection indicates a system failure but the system is actually good (working). Also, an inspection may report that the system is good when in fact it is failed; this is a false negative. The corresponding probabilities of a false positive, and a false negative at an inspection are denoted by $\alpha$, and $\beta$ respectively. We assume that, when an inspection is positive, the system is replaced. Thus the system is replaced at a true positive inspection, in error at a false positive inspection, and at $M T$, whichever occurs first.

One might envisage circumstances in which at a false positive a deeper investigation of the state of the system is carried out to reveal the true system state. Such a secondary inspection would come at an extra cost; but because it would ultimately reveal the system state, in such circumstances false positives would not occur, and a positive inspection would merely imply an additional cost. Thus, we do not consider this scenario. We emphasise that, in the model we develop in this paper, at a positive inspection, the system is replaced. In a real context, a maintainer may not have the capability to carry out the secondary inspection; the maintainer responds to a positive inspection (whether true or false) with an immediate replacement. This response is referred to as in-house maintenance by Berrade et al. [12].

We assume replacement renews the system so that conceptually the system comprises a component located in a socket which together perform an operational function (Ascher and Feingold [19]). In this way, by replacement we mean the replacement of the component in the socket with another functionally new component from a common stockpile of components.

Further, we suppose that, when the component fails, the system fails. The lifetime $X$ of a component has a general distribution $F$ with corresponding reliability function $R$. In our numerical study in Section 3, we suppose that $F$ may be a mixture, although in the determination of our results the exact nature of $F$ does not need to be specified. In its simplest form, in our first example, the mixture we consider is a mixture of two sub-distributions: one that represents components with relatively short lives, and one that represents components with long lives. A short component life may be the result of using a poor quality spare part, or of poor quality installation. Either way, this is conceptually what we mean by poor quality replacement. This idea has been studied in a reliability context (e.g. [20]), and a maintenance context (e.g. Scarf et al. [21]). Furthermore, and more generally in 
our second example, we consider a continuous mixture model for the lifetimes of a heterogeneous population of components. Overall, in the numerical study, our purpose is to illustrate the effect of poor quality inspection and component heterogeneity, or poor component quality, upon a preventive maintenance policy.

Regarding the costs, we suppose that the preventive cost $c_{m}$ is smaller than the corrective cost $c_{r}$, even though the component is replaced in both cases. We justify this assumption on the basis that, on replacement, components may be re-conditioned and returned to inventory. In the case of a false positive or a preventive replacement at $M T$, little reconditioning may be required. In the case of a true positive, an overhaul of the component may be required. Furthermore, it is worth noting that, as the system is a protection system, $c_{r}$ is associated with the failure of the protection system, and not the protected system. Thus, failure of the protection system only has an impact on the protected system if the protected system demands protective action from the protection system when the protection system is in the failed state. Such an event is modelled through the cost of unavailability $c_{d}$, which is interpreted as the product of notional quantities: the expected cost per unmet demand (for the operational function of the protection system), and the rate of occurrence of unmet demands.

\section{A. Some characterisations of the optimal policy}

The random variables $K_{1}$ and $K_{2}$ take values in $0,1,2, \ldots M$.

Now

$$
P\left(K_{1}=0\right)=1-R(T),
$$

and for $i=1,2, \ldots M-1$

$$
\begin{aligned}
& P\left(K_{1}=i\right)= \\
& (R(i T)-R((i+1) T))(1-\alpha)^{i}+R(i T)(1-\alpha)^{i-1} \alpha,
\end{aligned}
$$

and

$$
P\left(K_{1}=M\right)=R(M T)(1-\alpha)^{M-1} .
$$

Its expected value is

$$
E\left[K_{1}\right]=\sum_{i=1}^{M} R(i T)(1-\alpha)^{i-1} .
$$

In the case that there is no inspection at $M T$, the expectation is

$$
E\left[K_{1}\right]=\sum_{i=1}^{M-1} R(i T)(1-\alpha)^{i-1} .
$$

For $K_{2}$, we have

$$
\begin{aligned}
& P\left(K_{2}=0\right)= \\
& \sum_{i=1}^{M-1} R(i T)(1-\alpha)^{i-1} \alpha+R(M T)(1-\alpha)^{M-1} ;
\end{aligned}
$$

and for $i=1,2, \ldots M-1$,

$$
\begin{aligned}
& P\left(K_{2}=i\right)= \\
& \sum_{k=1}^{M-i}(R((k-1) T)-R(k T))(1-\alpha)^{k-1} \beta^{i-1}(1-\beta)+ \\
& (R((M-i) T)-R((M-i+1) T))(1-\alpha)^{M-i} \beta^{i-1},
\end{aligned}
$$

and

$$
\begin{gathered}
P\left(K_{2}=M\right)=(1-R(T)) \beta^{M-1} . \\
E\left[K_{2}\right]= \\
\sum_{i=1}^{M}(R((i-1) T)-R(i T))(1-\alpha)^{i-1} \frac{1-\beta^{M+1-i}}{1-\beta} .
\end{gathered}
$$

If there is no inspection at $M T$, the expectation is

$E\left[K_{2}\right]=\sum_{i=1}^{M-1}(R((i-1) T)-R(i T))(1-\alpha)^{i-1} \frac{1-\beta^{M-i}}{1-\beta}$.

Therefore, the expected length of a cycle is

$$
\begin{aligned}
& E[\tau]=E\left[\left(K_{1}+K_{2}\right) T\right]= \\
& \sum_{i=1}^{M}(1-\alpha)^{i-1} T \frac{\beta^{M+1-i}-\beta}{1-\beta} R(i T)+ \\
& \sum_{i=1}^{M}(1-\alpha)^{i-1} T \frac{1-\beta^{M+1-i}}{1-\beta} R((i-1) T) .
\end{aligned}
$$

The expected uptime in a cycle, $E[U]$, is

$$
\begin{aligned}
& E[U]=\sum_{i=1}^{M}(1-\alpha)^{i-1} \int_{(i-1) T}^{i T} t d F(t)+ \\
& \sum_{i=1}^{M-1} i T \alpha(1-\alpha)^{i-1} R(i T)+M T(1-\alpha)^{M-1} R(M T)= \\
& \sum_{i=1}^{M}(1-\alpha)^{i-1} \int_{(i-1) T}^{i T} R(t) d t .
\end{aligned}
$$

In addition, the expected downtime in a cycle is

$$
E[D]=E[\tau]-E[U] .
$$

Regarding costs, the expected cost derived from inspections, $c_{i n s}$, is

$$
c_{i n s}=c_{0}\left(E\left[K_{1}\right]+E\left[K_{2}\right]\right) .
$$

Therefore,

$$
\begin{aligned}
& c_{i n s}= \\
& c_{0} \sum_{i=1}^{M} R(i T)(1-\alpha)^{i-1}+ \\
& c_{0} \sum_{i=1}^{M}(R((i-1) T)-R(i T))(1-\alpha)^{i-1} \frac{1-\beta^{M+1-i}}{1-\beta} .
\end{aligned}
$$

The system is replaced at a false positive, or when it is detected to be failed (true positive), or preventively at $M T$, whichever occurs first. Both a false positive and the preventive maintenance at $M T$ incur a cost $c_{m}$. The associated cost due 
to the replacement of a failed system is $c_{r}$. Hence, the cost derived from the replacement of the system is

$$
\left\{\begin{array}{l}
c_{r}, \quad \text { if } X<M T \text { and there are no false positives, } \\
c_{m}, \quad \text { otherwise, }
\end{array}\right.
$$

with

$$
\begin{aligned}
& P\left(c_{r}\right)=\sum_{i=1}^{M}(1-\alpha)^{i-1} \int_{(i-1) T}^{i T} d F(t)= \\
& 1-\alpha \sum_{i=1}^{M-1} R(i T)(1-\alpha)^{i-1}-(1-\alpha)^{M-1} R(M T),
\end{aligned}
$$

and

$$
\begin{aligned}
& P\left(c_{m}\right)= \\
& \alpha \sum_{i=1}^{M-1} R(i T)(1-\alpha)^{i-1}+(1-\alpha)^{M-1} R(M T),
\end{aligned}
$$

where $P\left(c_{r}\right)$, and $P\left(c_{m}\right)$ denote the probabilities that the replacement cost is $c_{r}$, and $c_{m}$ respectively. The expected cost derived from the replacement of the system is

$$
\begin{aligned}
& c_{r e n}= \\
& \left(c_{m}-c_{r}\right) \alpha \sum_{i=1}^{M-1} R(i T)(1-\alpha)^{i-1}+ \\
& \left(c_{m}-c_{r}\right)(1-\alpha)^{M-1} R(M T)+c_{r} .
\end{aligned}
$$

The expected total cost incurred in a cycle is then

$$
\begin{aligned}
& C(\tau)=c_{i n s}+c_{r e n}+c_{d} E[D]=c_{i n s}+c_{r e n}+ \\
& c_{d}\left(E[\tau]-\sum_{i=1}^{M}(1-\alpha)^{i-1} \int_{(i-1) T}^{i T} R(t) d t\right) .
\end{aligned}
$$

Therefore, the cost-rate (the long-run cost per unit time) turns out to be

$$
\begin{aligned}
& Q(T, M)=c_{d}+ \\
& \frac{c_{\text {ins }}+c_{r e n}-c_{d}\left(\sum_{i=1}^{M}(1-\alpha)^{i-1} \int_{(i-1) T}^{i T} R(t) d t\right)}{E[\tau]},
\end{aligned}
$$

with $E[\tau]$ given in (3). In the case that $M=1$, the expression in (6) is

$$
\begin{aligned}
& Q(T, M)= \\
& c_{d}+\frac{c_{0}+\left(c_{m}-c_{r}\right) R(T)+c_{r}-c_{d} \int_{0}^{T} R(t) d t}{T} .
\end{aligned}
$$

The next lemma gives two limits for $Q(T, M)$ which are the key in the proofs of the results concerning the existence of a finite optimum policy $T$.

Lemma 1: Given $M, Q(T, M)$ is a continuous function so that

$\lim _{T \rightarrow 0} Q(T)=\infty$ and $\lim _{T \rightarrow \infty} Q(T)=c_{d}$.

The next result provides a sufficient condition to ensure the existence of a finite optimum $T^{\star}$ for a given $M$ when $c_{m}=c_{r}$.

Proposition 1: Suppose that $c_{m}=c_{r}$, and the number of inspections previous to the preventive maintenance, $M$, is fixed. If there exists some $T>0$ satisfying one of two conditions

a) $\quad T R(M T)>$

$$
\begin{aligned}
& \frac{c_{0}}{c_{d}(1-\beta)}\left(1-\frac{\beta^{M+1}-\beta(1-\alpha)^{M}}{\beta-(1-\alpha)} \frac{\alpha}{1-(1-\alpha)^{M}}\right) \\
& +\frac{\alpha c_{r}}{c_{d}\left(1-(1-\alpha)^{M}\right)}
\end{aligned}
$$

if $\alpha+\beta \neq 1$, or

$$
\begin{aligned}
& T R(M T)> \\
& \frac{c_{0}}{c_{d}(1-\beta)}\left(1-\frac{M \beta^{M}(1-\beta)}{1-\beta^{M}}\right)+\frac{c_{r}(1-\beta)}{c_{d}\left(1-\beta^{M}\right)}
\end{aligned}
$$

if $\alpha+\beta=1$,

then there exists a finite $T^{\star}$ minimizing $Q(T, M)$ in (6).

Lemma 2: The following properties apply.

i) $\max \left(c_{r}, c_{m}\right) \geq c_{r e n} \geq \min \left(c_{r}, c_{m}\right)$.

ii) $E[U]<\mu \frac{1-(1-\alpha)^{M}}{\alpha}$ for a given $M$.

The next results give some sufficient conditions under which the optimum inspection interval, $T^{\star}$, does not exist when the number of inspections $M$ is fixed.

Proposition 2: Given a fixed $M$, if $\mu=E[X]$ satisfies

$$
\mu \leq \frac{\alpha\left(c_{0}+\min \left(c_{r}, c_{m}\right)\right)}{c_{d}\left(1-(1-\alpha)^{M}\right)},
$$

then $T^{\star}=\infty$.

Condition (9) broadly means that, if maintenance costs are large or the false positive probability is large or both, then the best policy is no inspection at all.

Theorem 1: Given a fixed $M \geq 1$, if

i) $c_{m} \leq c_{r}$, and

$c_{0}\left(\frac{1-\beta^{M}}{1-\beta}+1-M\right)+c_{m} \geq c_{d} \mu \frac{1-(1-\alpha)^{M}}{\alpha}$; or

ii) $c_{m}>c_{r}$, and

$c_{0}\left(\frac{1-\beta^{M}}{1-\beta}+1-M\right)+c_{r} \geq c_{d} \mu \frac{1-(1-\alpha)^{M}}{\alpha}$

then $T^{\star}=\infty$.

Corollary 1: Given a fixed $M>1$, let $\beta_{M 0}$ be defined as

$$
\beta_{M 0}=\min \left\{\beta \mid \frac{1-\beta^{M}}{1-\beta} \geq v\left(\mu, M, \alpha, c_{0}, c_{r}, c_{m}, c_{d}\right)\right\}
$$

where

$$
\begin{aligned}
& v\left(\mu, M, \alpha, c_{0}, c_{r}, c_{m}, c_{d}\right)= \\
& \frac{c_{d} \mu \frac{1-(1-\alpha)^{M}}{\alpha}-\min \left(c_{r}, c_{m}\right)}{c_{0}}+M-1 .
\end{aligned}
$$

If $\beta \geq \beta_{M 0}$, then $T^{\star}=\infty$.

Note that Corollary 1 only makes sense when $M>1$; that is, when there are several inspections, and the inspection may fail to detect a failure, only then does a condition on $\beta$ exist. Thus, if one insists on conducting an inspection, if $\beta$ is (sufficiently) large, then such an inspection should be postponed indefinitely.

Next, we aim to obtain a condition for the existence of an optimum number of inspections, $M^{\star}$, and hence for preventive replacement to be optimal when the time to failure follows 
an exponential distribution. In general, $Q\left(T, M^{\star}\right)$ has a local minimum at $M^{\star}$ if

$$
Q\left(T, M^{\star}+1\right) \geq Q\left(T, M^{\star}\right), \quad Q\left(T, M^{\star}-1\right)>Q\left(T, M^{\star}\right) .
$$

Theorem 2: If the time to failure is an exponential distribution, and $\beta=0$, then, for any given $T, M^{\star}=\infty$.

The result is not necessarily true in the case of $\beta \neq 0$, as the following counter examples establish. With costs $c_{0}=1$, $c_{m}=5, c_{r}=10, c_{d}=5000, \alpha=0$, and an exponential time to failure distribution, we obtain

- $\lambda=100, \beta=0.8, M^{\star}=1, T^{\star}=0.0063, Q\left(T^{\star}, M^{\star}\right)=$ 2613.751 .

- $\lambda=1, \beta=0.9, M^{\star}=1, T^{\star}=0.0498, Q\left(T^{\star}, M^{\star}\right)=$ 247.82 .

It is known (Barlow and Proschan [22]) that, when the failures of a system are revealed as soon as they occur, and the time to failure follows an exponential distribution, then the lack of memory property implies that the best policy is no preventive maintenance $\left(M^{\star}=\infty\right)$. Theorem 2 indicates that this classic result still holds when the inspection procedure is only subject to false positives. Thus, there is no need to replace the system before it fails provided that the system in use is as-good-as-new, and the maintainer can be confident that a potential failure will be detected in the next inspection. When false negatives can occur, $M^{\star}=\infty$ may not be the best policy. The examples show that, although there is nothing to be gained from preventive replacement of a nonageing system if it is known to be in the good state, if it is not known to be in the good state (because inspections are subject to false negatives) it may be worthwhile to carry out preventive replacement at inspection. This condition is true because replacement guarantees the restoration of system functionality whereas inspection does not. Thus, and this is the crux of our work here, when a protection or cold-standby system has an exponential time to failure, and inspections are subject to false negatives, then contrary to the classic result, preventive replacement may indeed be cost-optimal. Thus, in summary, Theorem 1 describes the consequences of low quality maintenance for an inspection policy when $M$, the number of inspections until preventive replacement, is given, and the distribution of the time to failure of the component has a general form. Theorem 2 shows that, when inspections of a protection or standby system are subject to false positives, the classic result (it is cost-sub-optimal to replace a non-ageing system) still holds; but when inspections are subject to false negatives, our examples show that the classic result is no longer valid.

\section{B. The operational reliability function}

In the context of perfect inspection, more frequent inspections will increase the operational reliability of the system while also increasing the cost-rate, so that a reliability or safety requirement will generally dominate policy choice (Scarf et al. [23]). With imperfect maintenance, it is possible to simultaneously maximise reliability and minimise cost (Scarf et al. [21]). Therefore, for the imperfect inspection policy that we consider in this paper, it is interesting to derive the operational reliability function. We do this now. Note that by the operational reliability function we mean the probability that the system is functioning at time $t$, and there has been no failure in $(0, t)$ given that the system was new at time $t=0$ (Lewis [24]). The terminology used here is the same as that in Christer [25] who formulate the reliability function for a delay-time model. The main difference is that, in [25], the inspection procedure is considered to be perfect, and has no effect on the reliability of the system.

Let $R_{T M}(t)$ denote the reliability function of the system under inspection every $T$ units of time and preventive replacement at $M T . R_{T M}(t)$ represents the probability that a system new at $t=0$ survives up to $t$, for all $t>0$. In addition, let $r_{T M}^{(m)}$ denote the reliability function for the system at time $t$ with $(m-1) T \leq t<m T$. It follows that

$$
r_{T M}^{(1)}(t)=R(t), \quad 0 \leq t<T
$$

is just the component reliability. When developing $r_{T M}^{(m)}$ for $m>1$, we have to take into account the possibility of a false positive at each inspection prior to the replacement at $M T$ of a non-failed system.

Therefore, for $T \leq t<2 T$,

$$
r_{T M}^{(2)}(t)=(1-\alpha) R(t)+\alpha R(T) r_{T M}^{(1)}(t-T) .
$$

For $2 T \leq t<3 T$, it follows that

$$
\begin{aligned}
& r_{T M}^{(3)}(t)=(1-\alpha)^{2} R(t)+ \\
& \alpha R(T) r_{T M}^{(2)}(t-T)+(1-\alpha) \alpha R(2 T) r_{T M}^{(1)}(t-2 T) .
\end{aligned}
$$

Let us now develop the expression of $r_{T M}^{(3)}(t)$ to explain its meaning. Replacing the corresponding expressions of $r_{T M}^{(2)}(t)$ and $r_{T M}^{(1)}(t)$, we obtain

$$
\begin{aligned}
& r_{T M}^{(3)}(t)= \\
& (1-\alpha)^{2} R(t)+\alpha R(T)(1-\alpha) R(t-T)+ \\
& \alpha^{2} R^{2}(T) R(t-2 T)+ \\
& (1-\alpha) \alpha R(2 T) R(t-2 T) .
\end{aligned}
$$

The first term represents the probability that the system, new at $t=0$, survives up to $t$ with no false positive either at $T$ or at $2 T$. The second term indicates that the system is renewed at $T$ due to a false positive. There is no false positive at $2 T$, and the system survives up to $t$. The third term corresponds to the case that there are two false positives at $T$ and $2 T$, and the component installed at $2 T$ survives to $t$. In the forth term, there is no false positive at $T$, but one occurs at $2 T$, and the system renewed at $2 T$ survives up to $t$.

For $3 T \leq t<4 T$,

$$
\begin{aligned}
& r_{T M}^{(4)}(t)=(1-\alpha)^{3} R(t)+ \\
& \alpha R(T) r_{T M}^{(3)}(t-T)+(1-\alpha) \alpha R(2 T) r_{T M}^{(2)}(t-2 T)+ \\
& (1-\alpha)^{2} \alpha R(3 T) r_{T M}^{(1)}(t-3 T) .
\end{aligned}
$$

The general formulation for $(m-1) T \leq t<m T$ is

$$
\begin{aligned}
& r_{T M}^{(m)}(t)=(1-\alpha)^{m-1} R(t)+ \\
& \sum_{i=1}^{m-1} \alpha(1-\alpha)^{i-1} R(i T) r_{T M}^{(m-i)}(t-i T) .
\end{aligned}
$$


For $M T \leq t<(M+1) T$,

$$
r_{T M}^{(M+1)}(t)=r_{T M}^{(M)}(M T) r_{T M}^{(1)}(t-M T) .
$$

For $k M T+(j-1) T \leq t<k M T+j T$ with

$k=0,1, \ldots$, and $j=1,2, \ldots, M$, the general formula turns out to be

$$
R_{T M}(t)=r_{T M}^{(k M+j)}(t)=\left(r_{T M}^{(M)}(M T)\right)^{k} r_{T M}^{(j)}(t-k M T) .
$$

Observe that there is no effect of false negatives on the operational reliability; these only affect availability.

\section{NUMERICAL EXAMPLES}

In this section, we present some results that aim to illustrate the mathematical dependence on the parameters of both the optimum inspection frequency and optimum number of inspections previous to the preventive maintenance.

\section{A. A discrete mixture failure time distribution}

The time to failure is assumed to be a mixture of two Weibull distributions. The failure rate of a Weibull distribution is given by

$$
r(t)=s \eta^{-s} t^{s-1}
$$

The reliability function of the Weibull mixture is

$$
R(t)=p e^{-\left(t / \eta_{1}\right)^{s_{1}}}+(1-p) e^{-\left(t / \eta_{2}\right)^{s_{2}}} .
$$

The characteristic lives, and shape parameters of the mixture are $\eta_{1}=500, \eta_{2}=7000$, and $s_{1}=2.5, s_{2}=4.5$, respectively. The sub-populations in the mixture verify that $r_{1}(t) \geq r_{2}(t)$, $t \geq 0$; that is, sub-population 1 is smaller than sub-population 2 in hazard rate order. This mixture is used to model early failures caused by a proportion $p$ of weak items represented by sub-population 1. For additional insight on stochastic orders, see Shaked and Shantikumar [26].

Parameter values in the example relate to a case study considered in Berrade et al. [12] regarding a protection device in a soft drink production process. The optimum policy, $T$ and $M$, for different values of the model parameters is contained in Table I. Table I shows that the inspection interval increases when the probability of false positives, $\alpha$, increases, whereas $T$ decreases when $\beta$ increases. Both results are as expected because of the risk of incurring unnecessary replacements in the first case, and to mitigate false negatives in the second.

Whenever $\eta_{1}, \eta_{2}, s_{1}, s_{2}$ increase, the inspection frequency is relaxed, and the optimum cost-rate decreases. When the proportion of weak items $p$ increases, more intensive inspection is required, and therefore the optimum cost-rate is also higher. The form of the cost-rate is illustrated in Fig. 1.

Fig. 2 illustrates the behaviour of the operational reliability. An inspection of Fig. 2 reveals interesting features. For small values of $p$, as in Fig. 2(a), $R_{T M}(t)>R(t)$. Thus, maintenance tends to increase the reliability. For large values of time $t$, the reliability is greater when inspection is carried out at $k T$ than when no inspection is carried out. When the proportion of weak items is high, the reliability function is not monotonic with $T$ as in Fig. 2 (b) and (c). That is, $R_{T M}(t)$ does not always increase when the inspection frequency increases. The high proportion of weak items $p=0.15$ and $p=0.3$ explains the non-monotonic behavior. The risk of replacement with a weak item is large; therefore, as $T$ decreases, the risk of a poor quality replacement increases.

Nevertheless, $R_{T M}(t)$ increases as $T$ decreases when the proportion of weak items is low as in Fig. 2(a) $(p=0.005)$. The same reason as before explains this fact: provided that the risk of low quality replacement is low, increasing the inspection frequency is beneficial from the reliability point of view. Moreover, when $p$ increases to very high values as in Fig. 2(c) $\left.(p=0.3), R_{T M}(t)\right)$ drops dramatically, and tends to be even below the reliability when no maintenance is carried out, (baseline reliability $R(t)$ ).

Fig. 3 illustrates the interaction effect between parameters. In Fig. 3(b), when $\alpha$ increases, the reliability decreases. The same argument as before applies here: the high probability of a low quality replacement $(p=0.15)$ after a false positive makes $R_{T M}(t)$ decrease. In Fig. 3(a), where the value of $p$ is small $(p=0.005)$, the behaviour is just the opposite: when $\alpha$ increases, so does the reliability.

\section{B. Continuous mixture failure time distribution}

Following Gupta and Gupta [27], we consider the following mixture.

Let $X$ be a continuous random variable with baseline failure rate $r(x)$, and $Z$ be a nonnegative random variable that models the environmental effect responsible for the heterogeneity in the population. The reliability function conditional upon $Z=$ $z$ is

$$
R(t \mid z)=e^{-z \int_{0}^{t} r(x) d x}
$$

and the conditional failure rate is

$$
r(t \mid z)=z r(t)
$$

In this case, a greater value of $z$ implies harder environmental conditions, and therefore $r\left(t \mid z_{1}\right) \geq r\left(t \mid z_{2}\right), z_{1}>z_{2}$.

The reliability function of the mixture, $R^{\star}(t)$, is expressed as

$$
R^{\star}(t)=\int_{0}^{\infty} g(z) e^{-z \int_{0}^{t} r(x) d x} d z,
$$

with $g(z)$ being the density function of $Z$.

In what follows, we assume the baseline failure rate corresponding to a Weibull distribution. Hence,

$$
R(t \mid z)=e^{-z(t / \eta)^{s}}
$$

and $Z$ is inverse Gaussian, so

$$
g(z)=\frac{1}{\left(2 \pi b z^{3}\right)^{\frac{1}{2}}} e^{-\frac{(d z-1)^{2}}{2 b z}}, \quad z, b, d>0 .
$$

It can be verified ( [27], [28]) that the failure rate of the mixture $r^{\star}(t)$ is

i) decreasing for $s \leq 1$,

ii) increasing $s \geq 2$, and

iii) non-monotonic of type $\mathrm{U}$ for $1<s<2$.

The form of $r^{\star}(t)$ of the type $U$ case implies that there exists a $t_{0}$ such that the derivative of $r^{\star}(t), r^{\star \prime}(t)$, is such that $r^{\star \prime}(t)>0$ for $t<t_{0}, r^{\star \prime}\left(t_{0}\right)=0, r^{\star \prime}(t)<0$ for $t>t_{0}$. 
Table II shows some results corresponding to the Weibullinverse Gaussian mixture.

Note that in all the cases where the mixture has a decreasing failure rate the corresponding $M^{\star}$ is not finite for moderate values of alpha and beta. This result makes sense provided that in both cases an improvement in the reliability of the system takes place as time goes by. However, we can see that, for $\beta$ large, or $\alpha$ large and $\beta \neq 0$, then $M^{\star} \neq \infty$; in fact, pure replacement $M=1$ is the best policy. In those cases such that $\beta=0$, then $M^{\star}=\infty$ no matter what the value of $\alpha$ is, and so we conjecture that the result in Theorem 2 also holds for DFR distributions.

When comparing cases 1 (IFR) and 3 (DFR) in Table II, both mixtures present the same mean time to failure. However, in the DFR case, the cost-rate is higher because in spite of the fact that the failure rate is decreasing it is initially high in comparison to the IFR case so that undetected early failures are likely.

Fig. 4 illustrates the point that if the mixture is DFR then the effect of increasing $\alpha$ is to decrease the reliability, while if the mixture is IFR then the effect of increasing $\alpha$ is to increase the reliability. Thus, if the mixture is DFR, increasing the inspection frequency can produce a negative effect on the reliability. The higher $\alpha$, the greater this negative effect will be. In addition, increasing $\alpha$ also increases the cost-rate. Note that the reliability function does not depend on the value of $\beta$.

\section{CONCLUSION}

This paper focuses on the effect of inspection errors and low quality maintenance when determining the optimal inspection interval and preventive maintenance time for a protection system or cold standby system in which failures are unrevealed. We develop a number of results that characterise the minimum cost-rate policy, and which allow us to quantify circumstances in which inspection is cost-inefficient.

When a safety requirement is prescribed, and it is therefore natural to consider the operational reliability of the system, false negatives affect availability ((3) and (4)), but not reliability. On the other hand, the effect of false positives on operational reliability is complex, and depends on the nature of the component reliability distribution. If this is IFR, then false positives increase operational reliability, so that false positives are a cost issue. For discrete mixtures, false positives provide also a positive effect on the reliability when the proportion of weak items is low. However, if the reliability distribution is not IFR, and replacement is imperfect (in the sense of Scarf et al. [2]), then the unnecessary replacements at false positives not only increase the cost-rate but may also decrease operational reliability and decrease availability. The same effect is detected when the proportion of weak items is high. This result is true because a new component introduced at replacement may be worse than the one in use.

When the system under inspection is DFR, and inspections are perfect, no preventive maintenance is recommended. However, we observe that this recommendation is no longer valid when false negatives can occur. If so, preventive maintenance can protect against the occurrence of a failure that remains undetected and causes unavailability and potential catastrophic failure of the protected system.

Thus, when one is dealing with a protection system, it would seem essential to inspect this system because inspection is the only way to know the state of the system. However, we show in this paper that, even if inspection is the only way to know the system state, and hence if the protection system is performing its required function (to protect a critical system), if maintenance is poorly executed (with high probability to commit mistakes at inspection and to introduce poor quality or badly installed components at preventive replacement), then it may be more prudent to do nothing. This kind of conclusion is not according to common engineering sense because in practice one might tend to act more frequently when an action is perceived to be only moderately effective.

\section{APPENDIX}

\section{PROOF OF LEMMA 1}

The cost-rate can be alternatively expressed as

$$
Q(T, M)=c_{d}+\frac{c_{0}}{T}+\frac{c_{r e n}-c_{d} E[U]}{T\left(E\left[K_{1}\right]+E\left[K_{2}\right]\right)}
$$

The expected uptime is given by

$$
E[U]=\int_{0}^{T} R(t) d t+\sum_{i=2}^{M}(1-\alpha)^{i-1} \int_{(i-1) T}^{i T} R(t) d t
$$

The second term in the previous expression is bounded as

$$
T \sum_{i=2}^{M}(1-\alpha)^{i-1} R(i T) \leq \sum_{i=2}^{M}(1-\alpha)^{i-1} \int_{(i-1) T}^{i T} R(t) d t,
$$

and

$$
\sum_{i=2}^{M}(1-\alpha)^{i-1} \int_{(i-1) T}^{i T} R(t) d t \leq T \sum_{i=2}^{M}(1-\alpha)^{i-1} R((i-1) T) .
$$

The upper, and lower bound of the foregoing inequalities tend to zero in both cases when $T$ tends to zero, and when $T$ tends to infinity (see Badía et al. [29]); and hence the following conditions hold.

$$
\lim _{T \rightarrow 0} E[U]=0 \text { and } \lim _{T \rightarrow \infty} E[U]=E[X]=\mu
$$

In addition,

$\lim _{T \rightarrow 0} c_{\text {ren }}=c_{m}, \quad \lim _{T \rightarrow \infty} c_{\text {ren }}=c_{r}$

$1 \leq K_{1}+K_{2} \leq M$. Hence $T \leq E\left[\left(K_{1}+K_{2}\right) T\right] \leq M T$, and the following two limits hold for a fixed $M$.

$\lim _{T \rightarrow 0} E\left[\left(K_{1}+K_{2}\right) T\right]=0$, and

$\lim _{T \rightarrow \infty} E\left[\left(K_{1}+K_{2}\right) T\right]=\infty$.

Thus the two limiting conditions in Lemma 1 are proved.

\section{PROOF OF PROPOSITION 1}

The total number of inspections in a cycle is bounded as follows.

$$
\begin{aligned}
& E\left[K_{1}\right]+E\left[K_{2}\right] \leq \sum_{i=1}^{M}(1-\alpha)^{i-1}\left(\frac{1-\beta^{M+1-i}}{1-\beta}\right)= \\
& \frac{1}{1-\beta}\left(\frac{1-(1-\alpha)^{M}}{\alpha}-\frac{\beta^{M+1}-\beta(1-\alpha)^{M}}{\beta-(1-\alpha)}\right)
\end{aligned}
$$


provided that $\alpha+\beta \neq 1$.

Next, a lower bound for the expected uptime is

$$
\begin{aligned}
& E[U]=\sum_{i=1}^{M}(1-\alpha)^{i-1} \int_{(i-1) T}^{i T} R(t) d t> \\
& \sum_{i=1}^{M}(1-\alpha)^{i-1} T R(i T)>\operatorname{TR}(M T) \frac{1-(1-\alpha)^{M}}{\alpha} .
\end{aligned}
$$

Then,

$$
\begin{aligned}
& c_{0}\left(E\left[K_{1}\right]+E\left[K_{2}\right]\right)+c_{r}-c d E[U]< \\
& \frac{c_{0}}{1-\beta}\left(\frac{1-(1-\alpha)^{M}}{\alpha}-\frac{\beta^{M+1}-\beta(1-\alpha)^{M}}{\beta-(1-\alpha)}\right)+ \\
& c_{r}-c_{d} T R(M T) \frac{1-(1-\alpha)^{M}}{\alpha} .
\end{aligned}
$$

In the case that $\alpha+\beta=1$, it follows that

$$
\begin{aligned}
& E\left[K_{1}\right]+E\left[K_{2}\right] \leq \sum_{i=1}^{M}(1-\alpha)^{i-1}\left(\frac{1-\beta^{M+1-i}}{1-\beta}\right)= \\
& \frac{1}{1-\beta}\left(\frac{1-\beta^{M}}{1-\beta}-M \beta^{M}\right) .
\end{aligned}
$$

Conditions (7) and (8) imply that

$$
c_{0}\left(E\left[K_{1}\right]+E\left[K_{2}\right]\right)+c_{r}-c_{d} E[U]<0
$$

for some $T>0$, and Lemma 1 leads to the result.

\section{PROOF OF LEMMA 2}

Result $i$ ) is true because

$0 \leq\left(\alpha \sum_{i=1}^{M-1} R(i T)(1-\alpha)^{i-1}+(1-\alpha)^{M-1} R(M T)\right)<1$,

and this follows because

$$
\begin{aligned}
& \alpha \sum_{i=1}^{M-1} R(i T)(1-\alpha)^{i-1}+(1-\alpha)^{M-1} R(M T)< \\
& \alpha \sum_{i=1}^{M-1}(1-\alpha)^{i-1}+(1-\alpha)^{M-1}= \\
& \alpha \frac{1-(1-\alpha)^{M-1}}{\alpha}+(1-\alpha)^{M-1}=1 .
\end{aligned}
$$

Result $i$ ) follows from the fact that

$$
\begin{aligned}
& \left(\sum_{i=1}^{M}(1-\alpha)^{i-1} \int_{(i-1) T}^{i T} R(t) d t\right) \leq \\
& \left(\sum_{i=1}^{M}(1-\alpha)^{i-1} \int_{0}^{\infty} R(t) d t\right)= \\
& \sum_{i=1}^{M}(1-\alpha)^{i-1} \mu=\mu \frac{1-(1-\alpha)^{M}}{\alpha} .
\end{aligned}
$$

\section{ProOF OF PROPOSITION 2}

$K_{1}$, and $K_{2}$ are $s$-dependent random variables such that $K_{1}+K_{2} \geq 1$, implying that $c_{i n s} \geq c_{0}$. Therefore, for a given $M$, condition (9) along with Lemma 2 imply that $Q(T, M) \geq$ $c_{d}$ for all $T>0$, and Lemma 1 leads to the result.

\section{PROOF OF THEOREM 1}

Let us analyze the numerator in the expression of the costrate given in (6) as follows.

$$
c_{i n s}+c_{r e n}-c_{d}\left(\sum_{i=1}^{M}(1-\alpha)^{i-1} \int_{(i-1) T}^{i T} R(t) d t\right)
$$

The cost derived from inspections in (5) can be alternatively written as

$$
\begin{aligned}
& c_{i n s}=c_{0}\left(\frac{1-\beta^{M}}{1-\beta}+\frac{\beta^{M}-\beta}{1-\beta} R(T)\right)+ \\
& c_{0} \sum_{i=2}^{M} R(i T)(1-\alpha)^{i-1}+ \\
& c_{0} \sum_{i=2}^{M}(R((i-1) T)-R(i T))(1-\alpha)^{i-1} \frac{1-\beta^{M+1-i}}{1-\beta} .
\end{aligned}
$$

Hence,

$$
c_{i n s} \geq c_{0}\left(\frac{1-\beta^{M}}{1-\beta}+\frac{\beta^{M}-\beta}{1-\beta} R(T)\right) .
$$

For a given $M \geq 1$, let us denote by $f(\beta)$ the function

$$
f(\beta)=\frac{\beta^{M}-\beta}{1-\beta} .
$$

$f(\beta)$ is a decreasing function, hence $f(\beta) \geq \lim _{\beta \rightarrow 1} f(\beta)=$ $1-M$, and it follows that

$$
c_{i n s} \geq c_{0}\left(\frac{1-\beta^{M}}{1-\beta}+1-M\right)
$$

provided that $f(\beta)$ is a negative function. Hence, if any of the conditions given in $i$ ) or $i i$ ) verify, then for all $M \geq 1$ and $T>0$, Lemma 2 implies that

$$
c_{i n s}+c_{r e n} \geq c_{d} E[U] .
$$

Therefore, for a given $M \geq 1, Q(T, M) \geq c_{d}$ for all $T>0$, and the result follows from Lemma 1 .

\section{ProOF OF COROLLARY 1}

$$
\begin{aligned}
& g(\beta, M)=\left(1-\beta^{M}\right) /(1-\beta) \text { is an increasing function with } \\
& \frac{\partial g(\beta, M)}{\partial \beta}=\frac{h(\beta)}{(1-\beta)^{2}},
\end{aligned}
$$

and $h(\beta)=1-M \beta^{M-1}(1-\beta)-\beta^{M}$ verifies that $h(0)=$ $1, \quad h(1)=0$, and $h^{\prime}(\beta)=-M(M-1) \beta^{M-2}(1-\beta)<0$. Therefore, $h(\beta) \geq 0$, and $g(\beta, M)$ is increasing with $\beta$. Hence, conditions $i$ ) or $i$ i) in Theorem 1 hold for $\beta \geq \beta_{M 0}$, and thus the result in Corollary 1 is obtained. 


\section{PROOF OF THEOREM 2}

If $\beta=0$, the cost-rate is

$$
Q(T, M)=c_{d}+\frac{c_{0}}{T}+\frac{a(T, M)}{b(T, M)}
$$

with $a(T, M)$, and $b(T, M)$ as

$$
\begin{aligned}
& a(T, M)=\left(c_{m}-c_{r}\right) \alpha \sum_{i=1}^{M-1} R(i T)(1-\alpha)^{i-1}+ \\
& \left(c_{m}-c_{r}\right)(1-\alpha)^{M-1} R(M T)+c_{r}- \\
& c_{d} \sum_{i=1}^{M}(1-\alpha)^{i-1} \int_{(i-1) T}^{i T} R(t) d t, \\
& b(T, M)=T \sum_{i=1}^{M}(1-\alpha)^{i-1} R((i-1) T) .
\end{aligned}
$$

In addition,

$$
\begin{gathered}
Q(T, M+1)=c_{d}+\frac{c_{0}}{T}+\frac{a(T, M+1)}{b(T, M+1)}, \\
a(T, M+1)=a(T, M)+g(T, M), \\
b(T, M+1)=b(T, M)+h(T, M)
\end{gathered}
$$

with

$$
\begin{aligned}
& g(T, M)= \\
& \left(c_{m}-c_{r}\right)(1-\alpha)^{M}(R((M+1) T)-R(M T))- \\
& c_{d}(1-\alpha)^{M} \int_{M T}^{(M+1) T} R(t) d t,
\end{aligned}
$$

and

$$
h(T, M)=T(1-\alpha)^{M} R(M T) .
$$

Then $Q(T, M+1) \leq Q(T, M)$ is equivalent to the inequality

$$
\frac{a(T, M)+g(T, M)}{b(T, M)+h(T, M)} \leq \frac{a(T, M)}{b(T, M)}
$$

which in turn is equivalent to

$$
\frac{g(T, M)}{h(T, M)} \leq \frac{a(T, M)}{b(T, M)}
$$

provided that $b(T, M) \geq 0$, and $h(T, M) \geq 0$.

The foregoing inequality can be also expressed as

$$
\begin{aligned}
& \frac{\left(c_{m}-c_{r}\right) R((M+1) T)-R(M T)}{R(M T)} \\
& -\frac{c_{d} \int_{M T}^{(M+1) T} R(t) d t}{R(M T)} \\
& \leq \\
& \frac{\left(c_{m}-c_{r}\right) \alpha \sum_{i=1}^{M-1} R(i T)(1-\alpha)^{i-1}}{\sum_{i=1}^{M}(1-\alpha)^{i-1} R((i-1) T)}+ \\
& \frac{\left(c_{m}-c_{r}\right)(1-\alpha)^{M-1} R(M T)+c_{r}}{\sum_{i=1}^{M}(1-\alpha)^{i-1} R((i-1) T)}- \\
& \frac{c_{d} \sum_{i=1}^{M}(1-\alpha)^{i-1} \int_{(i-1) T}^{i T} R(t) d t}{\sum_{i=1}^{M}(1-\alpha)^{i-1} R((i-1) T)} .
\end{aligned}
$$

If the time to failure follows an exponential distribution with rate $\lambda=1 / \mu$, then we get

$$
\begin{aligned}
& \left(\alpha \sum_{i=1}^{M-1}(1-\alpha)^{i-1} R(i T)+(1-\alpha)^{M-1} R(M T)\right)= \\
& \frac{\alpha e^{-\lambda T}+(1-\alpha)^{M} e^{-\lambda M T}\left(1-e^{-\lambda T}\right)}{1-(1-\alpha) e^{-\lambda T}}
\end{aligned}
$$

and

$$
\sum_{i=1}^{M}(1-\alpha)^{i-1} R((i-1) T)=\frac{1-(1-\alpha)^{M} e^{-\lambda M T}}{1-(1-\alpha) e^{-\lambda T}} .
$$

Also,

$$
\begin{aligned}
& \sum_{i=1}^{M}(1-\alpha)^{i-1} \int_{(i-1) T}^{i T} R(t) d t= \\
& \frac{\left(1-e^{-\lambda T}\right)\left(1-(1-\alpha)^{M} e^{-\lambda M T}\right)}{\lambda\left(1-(1-\alpha) e^{-\lambda T}\right)} .
\end{aligned}
$$

Then, the inequality in (10) is given by

$$
\begin{aligned}
& \left(c_{m}-c_{r}\right)\left(e^{-\lambda T}-1\right) \leq \\
& \frac{\left(c_{m}-c_{r}\right)\left(\alpha e^{-\lambda T}+(1-\alpha)^{M} e^{-\lambda M T}\left(1-e^{-\lambda T}\right)\right)}{1-(1-\alpha)^{M} e^{-\lambda M T}}+ \\
& \frac{c_{r}\left(1-(1-\alpha) e^{-\lambda T}\right)}{1-(1-\alpha)^{M} e^{-\lambda M T}} .
\end{aligned}
$$

Given that $1-(1-\alpha)^{M} e^{-\lambda M T} \geq 0$ for all $T$, the previous inequality is in turn equivalent to

$$
c_{m}\left(e^{-\lambda T}-1\right) \leq c_{m} \alpha e^{-\lambda T},
$$

which is always true for all $M$ and $T$ as the left hand side of the previous expression is less than or equal to zero.

Note that, if $\alpha=0$, then the last inequality also holds and proves the result for perfect inspections (Barlow and Proschan [22]).

Therefore, under the conditions of Theorem 2, $Q(T, M+1)<$ $Q(T, M)$ for all $M$, and the result holds. This completes the proof.

\section{ACKNOWLEDGMENT}

The work of M.D. Berrade has been supported by both the Spanish Ministry of Science and Innovation, and the Spanish Ministry of Economy and Competitiveness under Projects MTM2010-15311 and MTM2012-36603-C02-02 respectively. The work of Cristiano A.V. Cavalcante has been supported by $\mathrm{CNPq}$ (Brazilian Research Council). The authors wish to thank an anonymous referee for their valuable comments.

\section{REFERENCES}

[1] G.E. Apostolakis and P.P Bansal, "Effect of human error on the availability of periodically inspected redundant systems," IEEE Transactions on Reliability, vol. 26, no. 3, pp. 220-225, 1977.

[2] P.A. Scarf and C.A.V. Cavalcante, "Modelling quality in replacement and inspection maintenance," International Journal of Production Economics, vol. 135, no. 1, pp. 372-381, Jan 2012. 
[3] A. Braasch, M. Althaus, and A. Meyna, "Influence of the mileage distribution on reliability prognosis models" in Safety, Reliability and Risk Analysis: Theory, Methods and Applications., Taylor and Francis, London. Martorell et al. (eds), pp. 2239-2244, 2008.

[4] R. Denning, "UK MOD approach to improving reliability", presented at IEC/TC56 Dependability Workshop, London, 1 edition, 2006.

[5] J.K. Vaurio, "Unavailability analysis of periodically tested standby components," IEEE Transactions on Reliability, vol. 44, no. 3, pp. 512\&, Sep. 1995

[6] S. Taghipour and D. Banjevic, "Periodic inspection optimization models for a repairable system subject to hidden failures," IEEE Transactions on Reliability, vol. 60, no. 1, pp. 275-285, Mar. 2011.

[7] S. Taghipour and D. Banjevic, "Optimal inspection of a complex system subject to periodic and opportunistic inspections and preventive replacements," European Journal of Operational Research, vol. 220, no. 3, pp. 649-660, Aug. 2012

[8] T. Tang, D. Lin, D. Banjevic and A.S.. Jardine, "Availability of a system subject to hidden failure inspected at constant intervals with non-negligible downtime due to inspection and downtime due to repair/replacement," Journal of Statistical Planning and Inference, vol. 143, no. 1, pp. 176-185, Jan. 2013.

[9] S. Okumura, A.K.S. Jardine and H. Yamashina, "An inspection policy for a deteriorating single-unit system characterized by a delay time model," International Journal of Production Research, vol. 34, no. 9, pp. 24412460, Sep. 1996.

[10] T.P McWilliams and H.F. Martz, "Human error considerations in determining the optimum test interval for periodically inspected standby systems," IEEE Transactions on Reliability, vol. 29, no. 4, pp. 305-310, 1980.

[11] M.D. Berrade, "A two-phase inspection policy with imperfect testing," Applied Mathematical Modelling, vol. 36, no. 1, pp. 108-114, Jan. 2012.

[12] M.D. Berrade, C.A.V. Cavalcante and P.A. Scarf, "Maintenance scheduling of a protection system subject to imperfect inspection and replacement," European Journal of Operational Research, vol. 218, no. 3, pp. 716-725, May 2012.

[13] M.D. Berrade, P.A. Scarf, C.A.V. Cavalcante and R.A. Dwight, "Imperfect inspection and replacement of a system with a defective state: A cost and reliability analysis," Reliability Engineering and System Safety, vol. 120, special issue, pp. 80-87, Dec. 2013.

[14] L. Gong, "The effect of testing errors on a repetitive testing process," European Journal of Operational Research, vol. 220, no. 1, pp. 115-124, Jul. 2012.

[15] Mike ten Wolde and Adel A. Ghobbar, "Optimizing inspection intervalsReliability and availability in terms of a cost model: A case study on railway carriers," Reliability Engineering and System Safety, vol. 114, pp. 137-147, Jun. 2013.

[16] R. Jiang and D.N.P. Murthy, "Modeling Failure-Data by Mixture of 2 Weibull Distributions: A Graphical Approach," IEEE Transactions on Reliability, vol. 44, no. 3, pp. 477-488, Sep. 1995.

[17] J.H. Cha and M. Finkelstein, "Stochastically ordered subpopulations and optimal burn-in procedure," IEEE Transactions on Reliability, vol. 59 no. 4, pp. 635-643, Dec. 2010.

[18] F. Flage, D.W. Coit, J.T. Luxhøj and T Aven, "Safety constraints applied to an adaptive Bayesian condition-based maintenance optimization model," Reliability Engineering \& System Safety, vol. 102, pp. 16-26, Jun. 2012.

[19] H. Ascher and H. Feingold, Repairable Systems Reliability. New York: Marcel Dekker, 1984

[20] R. Jiang and D.N.P. Murthy, "Mixtures of weibull distributionsparametric characterization of failure rate function," Applied Stochastic Models and Data Analysis, vol. 14, no. 1, pp. 47-65, Mar. 1998.

[21] P.A. Scarf, C.A.V. Cavalcante, R. Dwight and P Gordon, "An age based inspection and replacement policy for heterogeneous components," IEEE Transactions on Reliability, vol. 58, no. 4, pp. 641-648, Dec. 2009.

[22] R.E. Barlow and F. Proschan, Mathematical Theory of Reliability. New York: Wiley, 1965.

[23] P.A. Scarf, R. Dwight and A. Al-Musrati, "On reliability criteria and the implied cost of failure for a maintained component," Reliability Engineering and System Safety, vol. 89, no. 2, pp. 199-207, Aug. 2005.

[24] E.E. Lewis, Introduction to Reliability Engineering. New York: Wiley, 1987.

[25] A.H. Christer, "Delay-Time Model of Reliability of Equipment Subject to Inspection Monitoring," Journal of the Operational Research Society, vol. 38, no. 4, pp. 329-334, Apr. 1987.

[26] M. Shaked, J.G. Shanthikumar, Stochastic Orders. Springer, 2007.
[27] P.L. Gupta and R.C. Gupta, "Ageing characteristics of the Weibull mixtures," Probability in the Engineering and Informational Sciences, vol. 10, no. 4, pp. 591-600, 1996.

[28] L.R. Jaisingh, D.K. Dey and W.S. Griffith, "Properties of a Multivariate survival distribution generated by a Weibull \& Inverse-Gaussian mixture," IEEE Transactions on Reliability, vol. 42, no. 4, pp. 618-622, Dec. 1993.

[29] F.G. Badía, M.D. Berrade and C.A. Campos, "Optimization of inspection intervals based on cost," Journal of Applied Probability, vol. 38, no. 4 pp. 872-881, Dec. 2001.

M.D. Berrade is a Lecturer in Statistics at the School of Engineering and Architecture of the University of Zaragoza. She obtained her Ph.D. from the University of Zaragoza for work on ageing properties and the maintenance of systems. She has published in Reliability Engineering and System Safety, Applied Mathematical Modelling, European Journal of Operational Research, Journal of Applied Probability, Applied Stochastic Models in Business and Industry, Probability in the Engineering and Informational Sciences, and others. Her current interests include inspection and maintenance of systems.

Philip A. Scarf is a Professor of Applied Statistics at the University of Salford. He obtained his Ph.D. in 1989 from the University of Manchester for work on the statistical modelling of corrosion. He has published more than 40 articles on the modelling of capital replacement, reliability, and maintenance in IEEE Transactions on Reliability, European Journal of Operational Research, Reliability Engineering and System Safety, Naval Research Logistics, Journal of the Operational Research Society, IMA Journal of Management Mathematics, International Transactions in Operations Research, and others. He chairs the IMA Conference on Modelling in Maintenance and Reliability; the $9^{t h}$ conference in the series will take place in 2016. He also has research interests in advanced robotics, extreme values, crack growth, and applications of operational research and statistics in sport. Prof. Scarf is a Fellow of the Royal Statistical Society of the UK, and a Fellow of The Institute of Mathematics and Its Applications (IMA) of the UK. He serves as Editor-inChief of the IMA Journal of Management Mathematics.

Cristiano A.V. Cavalcante is Lecturer in Operational Research and Logistics at the Universidade Federal de Pernambuco (UFPE), at the Management Engineering Department. He obtained his Ph.D in 2005 from the Universidade Federal de Pernambuco for work on Multicriteria Decision Models to Support Maintenance Problems. He has published more than 20 articles in Reliability Engineering and System Safety, European Journal of Operational Research, IEEE Transactions on Reliability, International Journal of Production Economics, IMA Journal of Management Mathematics, Journal of Quality in Maintenance Engineering, Mathematical Problems in Engineering, International Journal of Advanced Manufacturing Technology, and others. His research interests include optimal maintenance, maintenance policies, and multi-criteria decision support. 
Table I: Discrete two component mixture; minimum cost-rate policy for various parameter values.

\begin{tabular}{|c|c|c|c|c|c|c|c|c|c|c|c|c|c|c|}
\hline \multirow[t]{2}{*}{ Case } & \multicolumn{5}{|c|}{$\begin{array}{l}\text { Mixed failure distribution } \\
\text { parameters }\end{array}$} & \multicolumn{4}{|c|}{ Cost parameters } & \multicolumn{2}{|c|}{$\begin{array}{l}\text { False -ve } \\
\text { and +ve } \\
\text { probabilities }\end{array}$} & \multicolumn{3}{|c|}{ Optimum policy } \\
\hline & $S_{1}$ & $\eta_{1}$ & $S_{2}$ & $\eta_{2}$ & $p$ & $c_{0}$ & $c_{\mathrm{m}}$ & $c_{\mathrm{r}}$ & $c_{D}$ & $\oint$ & $\alpha$ & $M^{*}$ & $T^{*}$ & $Q^{*}$ \\
\hline 1 & 2.5 & 500 & 4.5 & 7000 & 0.1 & 5 & 55 & 105 & 1.35 & 0.2 & 0.2 & 7 & 606 & 0.067 \\
\hline 2 & 2.5 & 500 & 4.5 & 7000 & 0.1 & 5 & 55 & 105 & 1.35 & 0.2 & 0.4 & 6 & 733 & 0.088 \\
\hline 3 & 2.5 & 500 & 4.5 & 7000 & 0.1 & 5 & 55 & 105 & 1.35 & 0.2 & 0.6 & 5 & 847 & 0.114 \\
\hline 4 & 2.5 & 500 & 4.5 & 7000 & 0.1 & 5 & 55 & 105 & 1.35 & 0 & 0 & 10 & 410 & 0.042 \\
\hline 5 & 2.5 & 500 & 4.5 & 7000 & 0.1 & 5 & 55 & 105 & 1.35 & 0 & 0.2 & 6 & 687 & 0.056 \\
\hline 6 & 2.5 & 500 & 4.5 & 7000 & 0.1 & 5 & 55 & 105 & 1.35 & 0.4 & 0.2 & 8 & 527 & 0.082 \\
\hline 7 & 2.5 & 500 & 4.5 & 7000 & 0.1 & 5 & 55 & 105 & 1.35 & 0.6 & 0.2 & 9 & 449 & 0.107 \\
\hline 8 & 1.5 & 500 & 4.5 & 7000 & 0.1 & 5 & 55 & 105 & 1.35 & 0.2 & 0.2 & 7 & 593 & 0.069 \\
\hline 9 & 3.5 & 500 & 4.5 & 7000 & 0.1 & 5 & 55 & 105 & 1.35 & 0.2 & 0.2 & 6 & 656 & 0.065 \\
\hline 10 & 2.5 & 400 & 4.5 & 7000 & 0.1 & 5 & 55 & 105 & 1.35 & 0.2 & 0.2 & 7 & 588 & 0.068 \\
\hline 11 & 2.5 & 600 & 4.5 & 7000 & 0.1 & 5 & 55 & 105 & 1.35 & 0.2 & 0.2 & 6 & 670 & 0.066 \\
\hline 12 & 2.5 & 500 & 3 & 7000 & 0.1 & 5 & 55 & 105 & 1.35 & 0.2 & 0.2 & 7 & 533 & 0.077 \\
\hline 13 & 2.5 & 500 & 6 & 7000 & 0.1 & 5 & 55 & 105 & 1.35 & 0.2 & 0.2 & 7 & 655 & 0.062 \\
\hline 14 & 2.5 & 500 & 4.5 & 5000 & 0.1 & 5 & 55 & 105 & 1.35 & 0.2 & 0.2 & 6 & 509 & 0.079 \\
\hline 15 & 2.5 & 500 & 4.5 & 9000 & 0.1 & 5 & 55 & 105 & 1.35 & 0.2 & 0.2 & 8 & 675 & 0.060 \\
\hline 16 & 2.5 & 500 & 4.5 & 7000 & 0.01 & 5 & 55 & 105 & 1.35 & 0.2 & 0.2 & 1 & 2557 & 0.029 \\
\hline 17 & 2.5 & 500 & 4.5 & 7000 & 0.05 & 5 & 55 & 105 & 1.35 & 0.2 & 0.2 & 5 & 740 & 0.051 \\
\hline 18 & 2.5 & 500 & 4.5 & 7000 & 0.25 & 5 & 55 & 105 & 1.35 & 0.2 & 0.2 & 11 & 469 & 0.114 \\
\hline 19 & 2.5 & 500 & 4.5 & 7000 & 0.1 & 2 & 55 & 105 & 1.35 & 0.2 & 0.2 & 7 & 588 & 0.062 \\
\hline 20 & 2.5 & 500 & 4.5 & 7000 & 0.1 & 10 & 55 & 105 & 1.35 & 0.2 & 0.2 & 6 & 686 & 0.074 \\
\hline 21 & 2.5 & 500 & 4.5 & 7000 & 0.1 & 5 & 55 & 70 & 1.35 & 0.2 & 0.2 & 7 & 608 & 0.065 \\
\hline 22 & 2.5 & 500 & 4.5 & 7000 & 0.1 & 5 & 55 & 140 & 1.35 & 0.2 & 0.2 & 7 & 605 & 0.069 \\
\hline 23 & 2.5 & 500 & 4.5 & 7000 & 0.1 & 5 & 55 & 105 & 0.8 & 0.2 & 0.2 & 6 & 724 & 0.053 \\
\hline 24 & 2.5 & 500 & 4.5 & 7000 & 0.1 & 5 & 55 & 105 & 2.2 & 0.2 & 0.2 & 8 & 510 & 0.085 \\
\hline
\end{tabular}

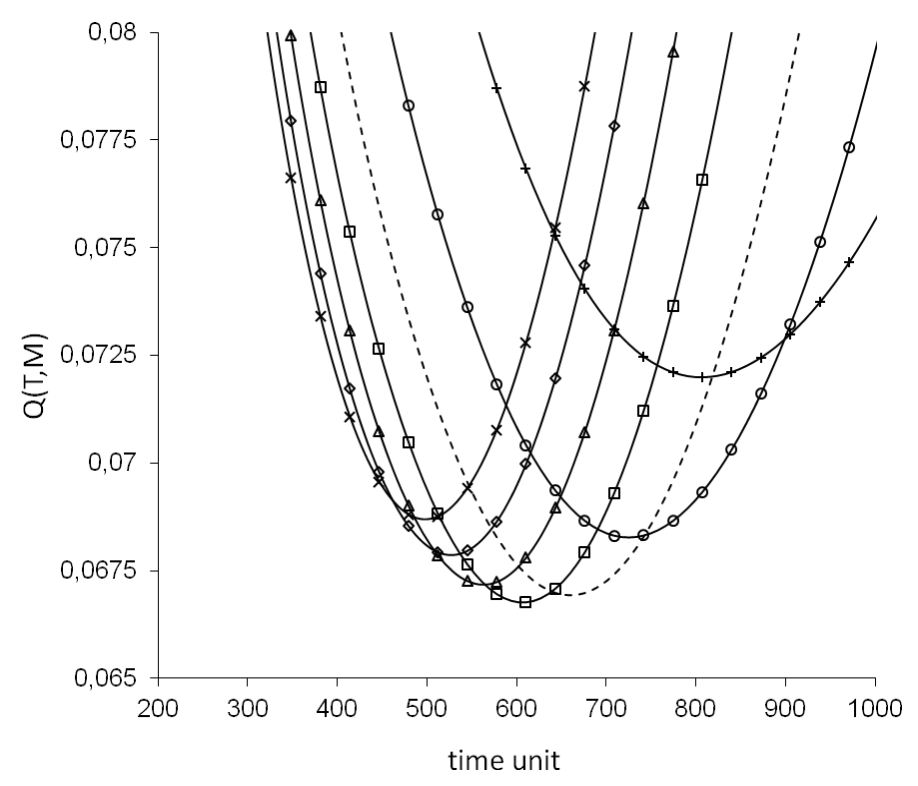

Figure 1. Cost-rate, $Q(T, M)$, as a function of $T$ for $M=4\left({ }^{-+}\right) ; M=5\left(-\mathrm{O}^{-}\right)$; $M=6(--) ; M=7\left(-\square^{-}\right) ; M=8\left(-\Delta^{-}\right) ; M=9\left(-{ }^{-}\right) ; M=10(-\mathrm{X}-)$.

Parameter values: $s_{1}=2.5, \eta_{1}=500, s_{2}=4.5, \eta_{2}=7000, p=0.10, \alpha=0.2, \beta=0.2$, $c_{0}=5, c_{m}=55 c_{r}=105, c_{D}=1.35$. 


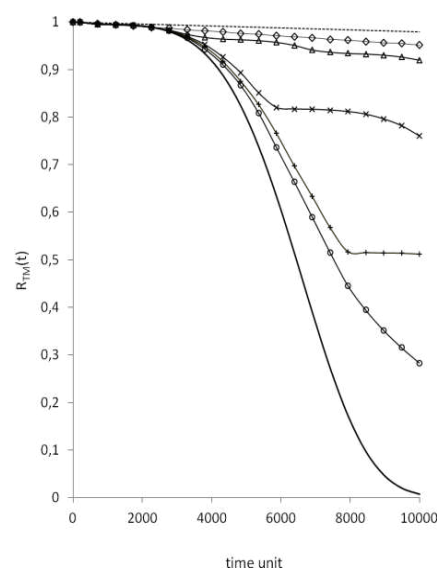

(a)

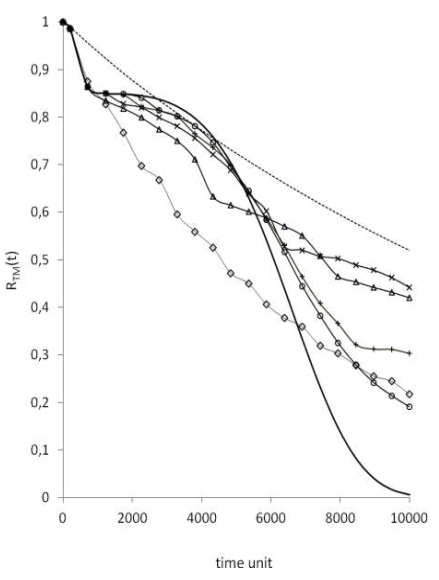

(b)

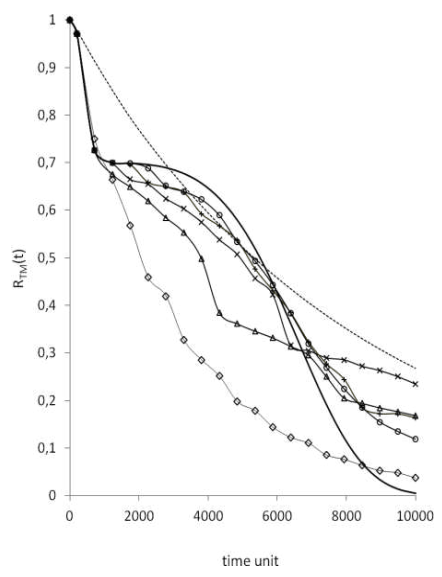

(c)

Figure 2. Reliability function $R_{T M}(t)$ as a function of $t$ for $\mathrm{M}=5$ and $\alpha=0.2$, for various values of $T$ :

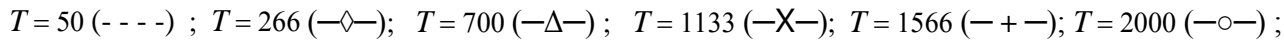
baseline reliability $R(t)$ (一). Discrete mixture (a) $p=0.005$; (b) $p=0.15$; (c) $p=0.3$; other parameter values: $s_{1}=2.5, \eta_{1}=500, s_{2}=4.5, \eta_{2}=7000$; costs as base case.

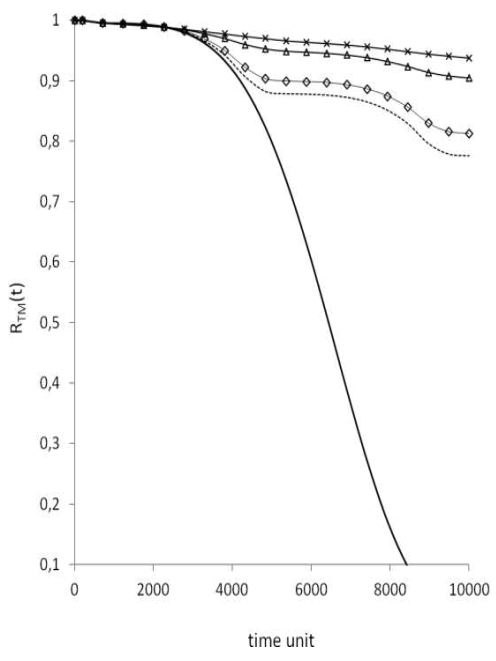

(a)

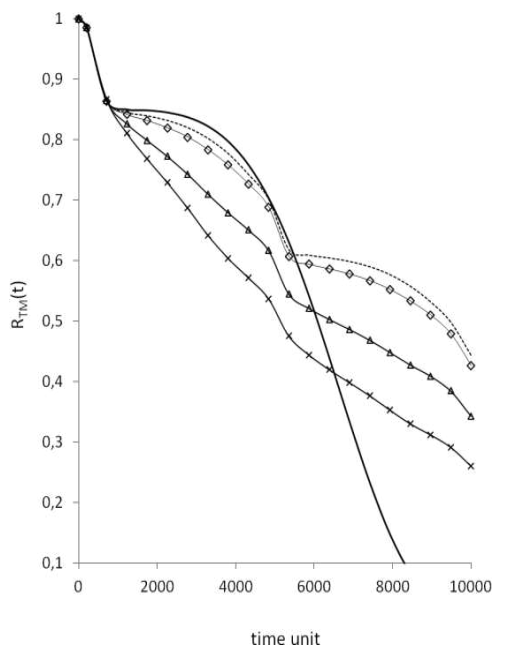

(b)

Figure 3. Reliability function $R_{T M}(t)$ as a function of $t$ for $M=7$ and $T=655$, for various values of $\alpha: \alpha=0.05$ $(---) ; \alpha=0.1(-\checkmark-) ; \alpha=0.3(-\Delta-) ; \alpha=0.5(-\mathrm{X}-)$; baseline reliability $R(t)(-)$. Discrete mixture: (a) $p=0.005$; (b) $p=0.15$. Other parameter values: $s_{1}=2.5, \eta_{1}=500, s_{2}=4.5, \eta_{2}=7000$; costs as base case. 
Table II: Continuous mixture: Weibull-inverse Gaussian. Minimum cost-rate policy for various parameter values.

\begin{tabular}{|c|c|c|c|c|c|c|c|c|c|c|c|c|c|}
\hline \multirow[t]{2}{*}{ Case } & \multicolumn{4}{|c|}{$\begin{array}{l}\text { Mixed failure } \\
\text { distribution } \\
\text { parameters }\end{array}$} & \multicolumn{4}{|c|}{ Cost parameters } & \multicolumn{2}{|c|}{$\begin{array}{l}\text { False -ve } \\
\text { and +ve } \\
\text { probabilities }\end{array}$} & \multicolumn{3}{|c|}{ Optimum policy } \\
\hline & $s$ & $\eta$ & $b$ & $d$ & $c_{0}$ & $c_{\mathrm{m}}$ & $c_{\mathrm{r}}$ & $c_{D}$ & $\oint$ & $\alpha$ & $M^{*}$ & $T^{*}$ & $Q^{*}$ \\
\hline 1 & 2 & 5008 & 1 & 1 & 5 & 55 & 105 & 1.35 & 0.2 & 0.2 & 2 & 731 & 0.076 \\
\hline 2 & 1.5 & 5008 & 1 & 1 & 5 & 55 & 105 & 1.35 & 0.2 & 0.2 & $\infty$ & 340 & 0.094 \\
\hline 3 & 1 & 2897 & 1 & 1 & 5 & 55 & 105 & 1.35 & 0.2 & 0.2 & $\infty$ & 272 & 0.157 \\
\hline 4 & 1 & 2897 & 1 & 1 & 5 & 55 & 105 & 1.35 & 0.4 & 0.2 & $\infty$ & 217 & 0.189 \\
\hline 5 & 1 & 2897 & 1 & 1 & 5 & 55 & 105 & 1.35 & 0.6 & 0.2 & 2 & 348 & 0.236 \\
\hline 6 & 1 & 2897 & 1 & 1 & 5 & 55 & 105 & 1.35 & 0.8 & 0.2 & 1 & 578 & 0.238 \\
\hline 7 & 1 & 2897 & 1 & 1 & 5 & 55 & 105 & 1.35 & 0.2 & 0.4 & $\infty$ & 339 & 0.198 \\
\hline 8 & 1 & 2897 & 1 & 1 & 5 & 55 & 105 & 1.35 & 0.2 & 0.6 & $\infty$ & 395 & 0.230 \\
\hline 9 & 1 & 2897 & 1 & 1 & 5 & 55 & 105 & 1.35 & 0.2 & 0.8 & 1 & 578 & 0.238 \\
\hline 10 & 1 & 2897 & 1 & 1 & 5 & 55 & 105 & 1.35 & 0.0 & 0.8 & $\infty$ & 529 & 0.218 \\
\hline 11 & 1 & 2897 & 1 & 1 & 5 & 55 & 105 & 1.35 & 0.0 & 0.6 & $\infty$ & 475 & 0.195 \\
\hline 12 & 1 & 2897 & 1 & 1 & 5 & 55 & 105 & 1.35 & 0.0 & 0.5 & $\infty$ & 444 & 0.182 \\
\hline 13 & 1 & 2897 & 1 & 1 & 5 & 55 & 105 & 1.35 & 0.0 & 0.4 & $\infty$ & 412 & 0.168 \\
\hline 14 & 2 & 5008 & 1 & 1 & 5 & 55 & 105 & 1.35 & 0.1 & 0.1 & 6 & 392 & 0.069 \\
\hline
\end{tabular}

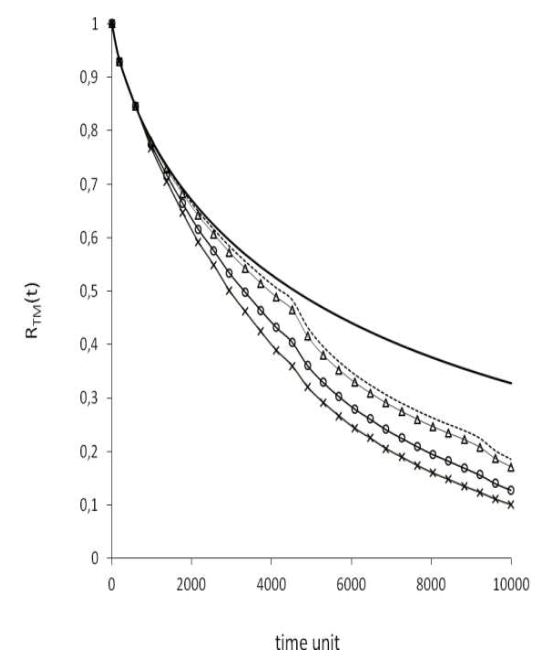

(a)

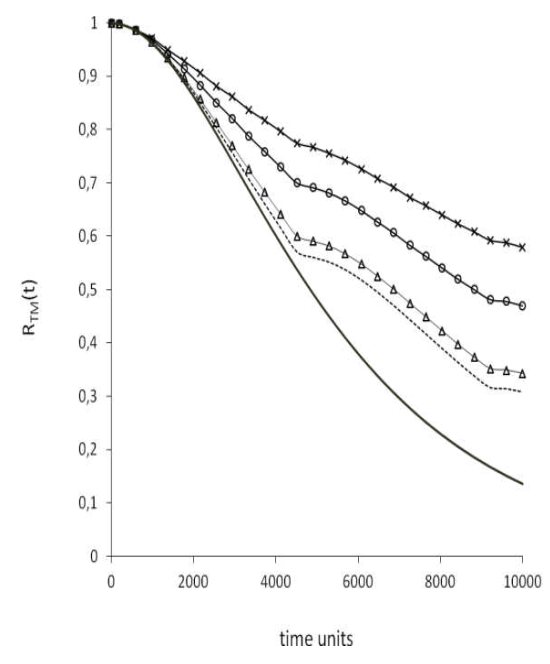

(b)

Figure 4. Reliability function $R_{T M}(t)$ as a function of $t$ for $M=7$ and $T=655$, for various values of $\alpha$ : $\alpha=0.05$ (- - ); $\alpha=0.1(-\Delta-) ; \alpha=0.3(-0-)$; and $\alpha=0.5$ (-X-) ; baseline reliability $R(t)(-)$.

Continuous mixture: (a) $s=0.8$ (DFR), (b) $s=2$ (IFR); other parameter values: $b=1 ; d=1, \eta=5008$; costs as base case. 